JOVAN P. ŠETRAJČIĆ' ${ }^{*}$, SINIŠA M. VUČENOVIĆ2, STEVO K. JAĆIMOVSKI ${ }^{3}$

${ }^{1}$ University of Novi Sad, Faculty of Sciences, Department of Physics, Novi Sad, Vojvodina, Serbia; ${ }^{2}$ University of Banja Luka, Faculty of Sciences, Physics, Banja Luka, Republic of Srpska, $B \& H ;{ }^{3}$ Academy of Criminology and Police Studies, Zemun, Serbia
Scientific paper

ISSN 0351-9465, E-ISSN 2466-2585

UDC: 666.652

doi:10.5937/ZasMat1602239S

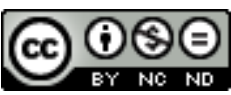

Zastita Materijala 57 (2) 239 - 243 (2016)

\title{
Possible states of charge carriers in thin multilayered superconductive ceramics
}

\begin{abstract}
This paper analyzes behavior (spectra and states) of the elementary charge carriers in anisotropic perovskite structures, such as modern superconducting ceramics. Translational symmetry of the atom (ion) distribution of the electron (or hole) system is broken by atomic/ionic/molecular sputtering and doping, as well as due to existence of two boundary surfaces. This is a charge carrier's model of high-temperature superconductors in which the observed symmetry breaking orthogonal to $\mathrm{CuO}$ planes was treated as a perturbation. The single-particle fermion's wave functions and the possible energies of charge carriers were determined.
\end{abstract}

Keywords: Charge carriers, boundaries, anisotropy, energy states and spectra, one-particle wave functions.

\section{INTRODUCTION}

The answer to the question of the oxide ceramics superconductivity mechanism must be undoubtedly sought in the phonon subsystem, in the elementary charges subsystem as well as in the interaction of these subsystems. With regard to the very anisotropic structure of the superconductive ceramics [1-4], we have attempted to construct a theoretical model conveying the broken translational symmetry of atoms (or molecules) arrangement along one direction in the crystal lattice, the difference of masses of these molecules and the presence of two boundary planes along this direction [5-9].

The phonon system is drawn out in this model [2,4-9]. We have determined the phonon states and their energy spectra and we have shown that, due to the broken crystal symmetry (actually because of deformed and tiny granular structure), the phonons of optical type owning the energy gaps are present here. The next task that we have attempted to solve is to determine and analyze the spectra of charge carriers (electrons or holes), Landau criterion, the probabilities of states and entropy within the same model [10-12].

${ }^{*}$ Corresponding author: Jovan P. Šetrajčić

E-mail: jovan.setrajcic@df.uns.ac.rs

Paper received: 09. 02. 2016.

Paper accepted: 24. 03. 2016.

Paper is available on the website:

www.idk.org.rs/journal

\section{FORMATION OF THE MODEL STRUCTURE}

In order to obtain Hamiltonian of the charge carriers in the structure with broken translational symmetry, it is the most suitable to start with the standard Hamiltonian of electron system in an ideal infinite structure:

$$
H_{i d}=\sum_{\vec{k}} \frac{\hbar^{2} k^{2}}{2 m^{*}} a_{\vec{k}}^{+} a_{\vec{k}},
$$

where $m^{*}$ is charge carriers effective mass, while $a_{\vec{k}}^{+}$and $a_{\vec{k}}$ are Fermi's creation and annihilation operators of charge carriers with momentum $\hbar \vec{k}$ and energy $\frac{\hbar^{2} k^{2}}{2 m^{*}}$ [13-15]. If we go over to the configuration space using the transformations:

$a_{\vec{n}}=\frac{1}{\sqrt{N}} \sum_{\vec{n}} a_{\vec{n}} e^{-i \vec{k} \vec{n}} ; \quad a_{\vec{k}}^{+}=\frac{1}{\sqrt{N}} \sum_{\vec{n}} a_{\vec{n}}^{+} e^{i \vec{k} \vec{n}}$,

where $N$ is the number of molecules in the considered structure, we get:

$$
H_{i d}=\sum_{\vec{n}} V a_{\vec{n}}^{+} a_{\vec{n}}-\sum_{\hat{n}, \vec{m}} W_{\vec{n} \vec{m}} a_{\vec{n}}^{+} a_{\vec{m}}
$$

where $\quad V=\frac{1}{N} \sum_{\vec{k}} \frac{\hbar^{2} k^{2}}{2 m^{*}} ; \quad W_{\vec{n} \vec{m}}=\frac{1}{N} \sum_{\vec{k}} \frac{\hbar^{2} k^{2}}{2 m^{*}} e^{i \vec{k}(\vec{n}-\vec{m})}$. Due to the canonicity of the transformation (2), the operators $a_{\vec{k}}^{+}$and $a_{\vec{k}}$ are also Fermi's operators. 
Let us recall the most important assumptions of our model: we consider the tetragonal, i.e. generalized cubic structure with very high anisotropy along the $z$ axis. It means that the lattice constant in this direction $\left(a_{z}\right)$ is a few times larger than the lattice constant $a_{x}, a_{y}$ in the directions $x$ and $y$. The translational symmetry is fully conserved in the $X Y$ planes, while the symmetry of the masses arrangement along the $z$ direction is broken (during the doping of the ceramic structure by the introducing of foreign atoms, the sputtered atoms locate along this direction because it is energetically most convenient). We also assume here that the structure under consideration is a thin film. It means that the components of lattice vector $\vec{n} \equiv\left(n_{x}, n_{y}, n_{z}\right)$ vary in the following way:

$n_{r} \in\left(-\frac{N_{r}}{2},+\frac{N_{r}}{2}\right) ; \quad r=(x, y) ; \quad n_{z} \in\left[0, N_{z}\right]$

The numbers of atoms $N_{x}$ and $N_{z}$ along the directions $x$ and $y$, respectively, may be indefinitely high, since we have the translational symmetry along these directions. The number of atoms along $z$ direction $\left(N_{z}\right)$ is limited. The above described model, i.e. the highly anisotropic matrix along the $z$ direction, necessarily doped with foreign atoms, can be used for getting some qualitative conclusions about the superconductive ceramics behavior. It is known [1-4] that the ceramic oxides are anisotropic along one privileged direction and that the superconductive state is realized by doping. But the real structure of the ceramic oxides - perovskites is approximated by the tetragonal structure. It is also assumed in the model that the sputtering is symmetric on the both of boundary planes: $n_{z}=0$ and $n_{z}=N_{z}$ and between the layers $n_{z}=0$ and $n_{z}=1$ (as well as between the layers $n_{z}$ $=N_{z}-1$ and $n_{z}=N_{z}$ ) $n_{0}$ foreign particles are placed, in such a way that the structure of the doped matrix is unchanged near the middle of the film.

If the behavior of the quantities from (3) may be expressed by the law:

$$
W_{\vec{n} \vec{m}}=\frac{W_{0}}{|\vec{n}-\vec{m}|^{h}} ; \quad W_{0}>0 ; \quad h>0,
$$

in the nearest-neighbors approximation we get:

$$
W_{n_{s} ; n_{s} \pm 1} \equiv W_{s}=\frac{W_{0}}{a_{s}^{h}} ; s=(x, y, z) .
$$

According to the described view of the doping, it is obvious that lattice constant $a_{z}$ in the doped structure becomes dependent on the position $n_{z}$, i.e. $a_{z} \rightarrow a_{z}\left(n_{z}\right)$. Because of the symmetry on the boundary planes, i.e. boundary layers: $a_{z}(0)=a_{z}\left(N_{z}\right)=\frac{a_{z}}{n_{0}+1} ; \quad a_{z}\left(N_{z} / 2\right)=a$, we may take:

$a_{z}\left(n_{z}\right)=a_{z}\left(1-\frac{n_{0}}{n_{0}+1} \beta_{z}^{2}\right) ; \quad \beta_{z}^{2} \equiv \frac{2 n_{z}}{N_{z}}-1$.

The dependence of the lattice constant on the index $n_{z}$ causes the dependence of the interaction along $z$ direction on the index $n_{z}$, i.e.

$W_{z} \rightarrow W_{z}\left(n_{z}\right)=$

$=\frac{W_{0}}{a_{z}^{h}\left(n_{z}\right)}=\frac{W_{0}}{a^{h}}\left(1-\beta_{z}^{2} \frac{n_{0}}{n_{0}+1}\right)^{-h} \approx W_{z}\left(1+\Phi \beta_{z}^{2}\right)^{\prime}$

where $\Phi=\frac{h n_{0}}{n_{0}+1}$. The interactions $W_{x}$ and $W_{y}$, according to the described picture, are unchanged. We must notice that the last two expressions are valid for even $N_{z}$. But, for large enough $N_{z}\left(N_{z} \approx\right.$ $\approx N_{z}+1$ ), or during the transition from $n_{z}$ to continual variable $z$, the deviations from the formulas (6) and (7) for odd $N_{z}$ are not essential. The values of $V$ are not dependent on the index of the site; because of they are unchanged during the doping. Hence we can write the Hamiltonian of the doped structure in the form:

$$
H=H_{B}+H_{V},
$$

$$
\begin{aligned}
H_{B} & =\sum_{n_{x}, n_{y}}\left\{a_{n_{x} n_{y} 0}^{+}\left[V a_{n_{x} n_{y} 0}-W_{x}\left(a_{n_{x}+1, n_{y} 0}+a_{n_{x}-1, n_{y} 0}\right)-W_{y}\left(a_{n_{x} n_{y}+1,0}+a_{n_{x} n_{y}-1,0}\right)-W_{z}(1-\Phi) a_{n_{x} n_{y} 1}\right]+\right. \\
& \left.+a_{n_{x} n_{y} N_{z}}^{+}\left[V a_{n_{x} n_{y} N_{z}}-W_{x}\left(a_{n_{x}+1, n_{y} N_{z}}+a_{n_{x}-1, n_{y} N_{z}}\right)-W_{y}\left(a_{n_{x} n_{y}+1, N_{z}}+a_{n_{x} n_{y}-1, N_{z}}\right)-W_{z}(1-\Phi) a_{n_{x} n_{y} N_{z}-1}\right]\right\}
\end{aligned}
$$

and, as we can see, it is related to the boundary layers $\left(n_{z}=0\right.$ and $\left.n_{z}=N_{z}\right)$, where obviously $W_{n_{x}, n_{y}, 0 ; n_{x}, n_{y},-1}=W_{n_{x}, n_{y}, N_{z} ; n_{x}, n_{y}, N_{z}+1}=0$, and for $H_{v}$ we find:

$$
\begin{aligned}
H_{v} & =\sum_{n_{x}, n_{y}} \sum_{z=0}^{N_{z}-1}\left\{a_{n_{x} n_{y} 0}^{+}\left[V a_{n_{x} n_{y} 0}-W_{x}\left(a_{n_{x}+1, n_{y} 0}+a_{n_{x}-1, n_{y} 0}\right)-W_{y}\left(a_{n_{x} n_{y}+1,0}+a_{n_{x} n_{y}-1,0}\right)-W_{z}(1-\Phi) a_{n_{x} n_{y} 1}\right]+\right. \\
& \left.+a_{n_{x} n_{y} N_{z}}^{+}\left[V a_{n_{x} n_{y} N_{z}}-W_{x}\left(a_{n_{x}+1, n_{y} N_{z}}+a_{n_{x}-1, n_{y} N_{z}}\right)-W_{y}\left(a_{n_{x} n_{y}+1, N_{z}}+a_{n_{x} n_{y}-1, N_{z}}\right)-W_{z}(1-\Phi) a_{n_{x} n_{y} N_{z}-1}\right]\right\} .
\end{aligned}
$$




\section{SINGLE-PARTICLE STATES}

We shall analyze the system described by Hamiltonian (8) using the orthonormalized singleelectron state functions:

$$
\begin{aligned}
& |\Psi\rangle=\sum_{n_{x}, n_{y}, n_{z}} A_{n_{x}, n_{y}, n_{z}} a_{n_{x}, n_{y}, n_{z}}^{+}|0\rangle ; \\
& \sum_{n_{x}, n_{y}, n_{z}}\left|A_{n_{x}, n_{y}, n_{z}}\right|^{2}=1 .
\end{aligned}
$$

We obtain the equations for finding the coefficient $A_{n_{x}, n_{y}, n_{z}}$ using the equations of motion for operators $a_{n_{x}, n_{y}, n_{z}}$. From the equation: $a_{n_{x}, n_{y}, n_{z}}(t)=a_{n_{x}, n_{y}, n_{z}}(0) e^{i \omega t}, \quad$ where $\omega=E / \hbar$, it follows:

$$
E a_{n_{x}, n_{y}, n_{z}}-\left[a_{n_{x}, n_{y}, n_{z}}, H\right] \equiv C_{n_{x} n_{y}, n_{z}} ; \quad C_{n_{x} n_{y}, n_{z}}=0 .(12)
$$

On the basis of equations $(8-10)$ and (12), we form operators: $C_{n_{x} n_{y}, 0}, C_{n_{x} n_{y}, N_{z}}$ and $C_{n_{x} n_{y}, n_{z}}$. After applying them to the functions (11) and using the general substitution:

$$
\alpha_{n_{x}, n_{y}, n_{z}}=\alpha_{n_{z}} e^{i\left(n_{x} a_{x} k_{x}+n_{y} a_{y} k_{y}\right)},
$$

where $k_{j}=\frac{2 \pi}{N_{j} a_{j}} v_{j} ; \quad j=(x, y) ; \quad v_{j} \in\left(-\frac{N_{j}}{2},+\frac{N_{j}}{2}\right)$ and on the basis of the fact that $V=2 \sum_{j=x, y, z} W_{j}$, we find the following system of $N_{z}+1$ difference equations for $n_{z}=0, \quad n_{z}=N_{z}$ and for $1 \leq n_{z} \leq N_{z}-1$, respectively:

$$
\begin{gathered}
\left(E-4 Q-2 W_{z}\right) A_{0}+W_{z}(1-\Phi) A_{1}=0, \\
\left(E-4 Q-2 W_{z}\right) A_{N_{z}}+W_{z}(1-\Phi) A_{N_{z}-1}=0, \\
\left(E-4 Q-2 W_{z}\right) \mathrm{A}_{n_{z}}+W_{z}\left(1+\Phi \beta_{z}^{2}\right)\left(\mathrm{A}_{n_{z}+1}+\mathrm{A}_{n_{z}-1}\right)=0 \\
\text { where } Q \equiv Q_{k_{x} k_{y}}=W_{x} \sin ^{2} \frac{a_{x} k_{x}}{2}+W_{y} \sin ^{2} \frac{a_{y} k_{y}}{2}
\end{gathered}
$$

We shall perform the further analysis in the continual approximation in order to avoid the complications arising during the determination of the coefficient $A_{n}$ from the system of difference equations (13). Introduction the continual variable $z$ through: $n_{z} \rightarrow z / a_{z}\left(N_{z} \rightarrow L / a_{z}\right)$ causes the following transformations of the expressions (7) and (6):

$$
\begin{aligned}
& a_{z ; n_{z}} \rightarrow a_{z}(z)=a_{z}\left[1-\frac{n_{0}}{n_{0}+1}\left(2 \frac{z}{L}-1\right)^{2}\right], \\
& W_{z ; n_{z}} \rightarrow W_{z}(z)=W_{z}\left[1+\Phi\left(2 \frac{z}{L}-1\right)^{2}\right] .
\end{aligned}
$$

The coefficients $\mathrm{A}_{n_{z}}$ will be transformed in the following way:

$$
\begin{gathered}
A_{n} \rightarrow A(z) ; \quad A_{n+1}+A_{n-1} \rightarrow A\left(z+\bar{a}_{z}\right)+A\left(z-\bar{a}_{z}\right) ; \\
A\left(z \pm \bar{a}_{z}\right) \approx A(z) \pm \bar{a}_{z} \frac{d A}{d z}+\frac{\bar{a}_{z}}{2} \frac{d^{2} A}{d z^{2}} \\
\bar{a}_{z} \equiv \bar{a}_{z}(z)=\frac{1}{L} \int_{0}^{L} d z a_{z}(z)=a_{z} \frac{2 n_{0}+3}{3\left(n_{0}+1\right)} .
\end{gathered}
$$

The important consequence of the transition to the continuum is the fact that the first two equations from (13) vanish from the calculation at $n_{z} \rightarrow z$, i.e. they are merged into the last of equations from (13), which in the continual approximation has the form:

$\frac{d^{2} A}{d z^{2}}+\frac{E-4 Q-\Phi\left(E-4 Q-2 W_{z}\right)\left(2 \frac{z}{L}-1\right)^{2}}{\bar{a}_{z}^{2}(z) W_{z}} A=0$

By the assumption:

$E>4 Q+2 W_{z} \equiv E_{x y z}^{(0)}$, and by the substitution: $\frac{2 z}{L}-1=\tau \zeta, \quad$ with $\quad \tau^{4}=\frac{W_{z}\left(\bar{a}_{z} L\right)^{2}}{4 \Phi\left(E-4 Q-2 W_{z}\right)}, \quad$ the equation (15) becomes known Hermite-Weber equation:

$$
\frac{d^{2} A}{d \zeta^{2}}+\left(\kappa-\zeta^{2}\right) A=0
$$

where $\quad \kappa=\frac{L}{2 \bar{a}_{z}} \frac{(E-4 Q)}{\sqrt{\Phi\left(E-E_{x y z}^{(0)}\right) W_{z}}}$. Here we introduce the requirement that the amplitudes $A$ are finite for arbitrary structure thickness (it means even for $L \rightarrow \infty$ ). For satisfying this requirement we must take known condition of the finiteness for the solutions for Hermite-Weber equation: $\kappa=2 \mu+1$; $\mu=0,1,2, \ldots$ On the basis of this we find:

$E_{1,2}=4 Q+2 b^{2}(2 \mu+1)^{2} \Phi W_{z}\left\{1 \pm\left[1-\frac{2}{(2 \mu+1)^{2} b^{2} \Phi}\right]^{1 / 2}\right\}$,

were $b=\bar{a}_{z} / L$. The expression for energies (17) indicates that index $\mu$ must be limited from below (the energies must be real):

$$
2 \mu \geq \frac{1}{b} \sqrt{\frac{2}{\Phi}}-1 .
$$

It means that the minimal allowed value of the index $\mu$ is the minimal integer which is bigger than 
the final term in (19). As we can see, the lower boundary of quantum number $\mu$ depends on the number of structural layers (through $N_{z}$ ), on the way of sputtering (through $n_{0}$ ) and on the type of ion-ion interaction (through $h$ ). If the thickness of the structure increases, the lower value of $\mu$ increases too.

For simplifying, instead of the expression (19), we will use the approximate expressions for energies, which we obtain by the expansion of the square root up to the quadratic terms:

$$
\begin{aligned}
& E_{1}=E_{x y z}^{(0)}+4 b^{2}(2 \mu+1)^{2} \Phi W_{z}-\frac{W_{z}}{2(2 \mu+1)^{2} b^{2} \Phi} ; \\
& E_{2}=E_{x y z}^{(0)}+\frac{W_{z}}{2(2 \mu+1)^{2} b^{2} \Phi} .
\end{aligned}
$$

It is very easy to notice that both obtained expressions for energies satisfy the necessary condition (18). However, by the analysis of (19), we can conclude the following.

- Since $E_{2}<E_{1}$, the states with energy $E_{2}$ are more stable and more populated and so they essentially define the normal behavior of the system.

- From the expressions (19) it follows that the increase of film thickness (the increase of $N_{z}$ ) causes the increase of lower boundary of the index $\mu$, and the correction of $E_{2}$, which depends on sputtering, decreases. This is in the complete agreement with the conclusions which we can accomplish without going over to continuum, i.e. directly analyzing discrete equations (13).

We can see in expressions defining $\zeta$, text under (18), that the boundaries of the interval for $\zeta$ are proportional to $L / \bar{a}_{z}=1 / b$ and so we can approximately take: $\zeta \in[-\infty,+\infty]$, where the approximation is better if the film is thicker. We can then express the solutions of equation (16) using Hermits polynomials:

$$
\begin{gathered}
A_{\mu}(\zeta)=\frac{e^{-\zeta^{2} / 2}}{\left(2^{\mu} \mu ! \sqrt{\pi}\right)^{1 / 2}} \mathrm{H}_{\mu}(\zeta) ; \\
\mathrm{H}_{\mu}(\zeta)=(-1)^{\mu} e^{\zeta^{2}} \frac{d^{\mu}}{d \zeta^{\mu}}\left(e^{-\zeta^{2}}\right) ; \quad \mu=0,1,2, \ldots
\end{gathered}
$$

In this way we have defined single-particle degenerate states of the system: for the wave functions - by the equations (11), (13) and (20) and for energies - by (19).

\section{CONCLUSION}

The particular features of high-temperature superconductors on the basis of oxide ceramics are their granular structure and the anisotropy of properties. The existence of the weak isotopic effect and Cooper pairs of charge carriers is experimentally verified, similar as in the conventional superconductors, but BCS model was not able to explain high critical temperature. For that reason and on the basis of established experimental results [16-20], we have proposed the model of ceramic structure as tetragonal i.e. generalized cubic structure in which interatomic distances along one direction are few times bigger than along other two directions. It is, energetically, most convenient if the sputtered atoms locate themselves just along this direction.

The analysis of phonon spectrum in our model [21-25] yields that we have phonon branches of optical type only in the spectrum (there exists energy gap). It means that for phonon excitation it is necessary that the energy (heat) is bigger than the energy gap.

The analysis of electron spectrum in these symmetrically deformed structures (with respect to the planes $n_{z}=0$ and $n_{z}=N_{z}$ ) yields that, as a consequence of existence of the boundaries along $z$ axes, we have two energy branches in the spectrum of charge carriers. Lower value of energy is related to more populated states and contains the term depending on the sputtering. This term decreases with increasing of the film thickness. Higher value of energy in the spectrum of charge carriers is not particularly analyzed because these levels are low populated.

\section{Acknowledgements}

This paper was partly financed by the Ministry of Education, Sciences and Technological Development of the Republic of Serbia (Grand Nos. ON171039 and TR-34019) and the Ministry of Science and Technology of the Republic of Srpska (Grant No: 19/6-020/961-23/14) as well as the Provincial Secretariat for Science and Technological Development of Vojvodina (Grant No: 114-451-927).

\section{REFERENCES}

[1] J.G.Bednorz, K.A.Müller (1987) Perovskite-Type Oxides - the New Approach to High-Tc Superconductivity, Nobel Lecture, Stockholm.

[2] J.P.Šetrajčić (2001) Superconductivity, Zmaj, Novi Sad.

[3] J.P.Šetrajčić (1998) Superconductivity and Fullerenes, Materials Science Forum, 282-283, 71-85.

[4] J.P.Šetrajčić, S.K.Jaćimovski, D.I.Raković, D.I.llić (2003) Phonon Spectra in Crystalline Nanostructures, in "Electrical and Computer Engineering Series:Advances in Simulation, Systems Theory and Systems Engineering", p.146-151, ISBN 9608052-70-X, Eds. N.E. Mastorakis, V.V. Kluev and Đ. Koruga, WSEAS Press, Athens 2003.

[5] J.P.Šetrajčić, B.S.Tošić, D.Lj.Mirjanić (1987) Superconductivity of One-Dimensional Metallic Structures, Physica A, 144, 353-364. 
[6] J.P.Šetrajčić, V.M.Zorić, N.V.Delić, D.Lj.Mirjanić, S.K. Jaćimovski (2011) Phonon Participation in Thermodynamics and Superconductive Properties of Thin Ceramic Films, Ch. 15, p. 317-348, In „Thermodynamics”, Ed. M. Tadashi, ISBN: 978-953307-544-0, InTech, Vienna 2011.

[7] B.S.Tošić, J.P.Šetrajčić, R.P.Djajić, D.Lj.Mirjanić (1987) Phonons in Broken-Symmetry Structures, Phys. Rev. B, 36, 9094-9097.

[8] J.P.Šetrajčić, R.P.Djajić, D.Lj.Mirjanić, B.S.Tošić (1990) Phonon Spectra in Superconducting, Ceramics Phys. Scr., 42, 732-741.

[9] S.K.Jaćimovki, J.P.Šetrajčić, M.S.Jaćimovski, V.V. Stojanović (2014) Phonon Contribution in Thermodynamics and Transport Properties of Ultrathin Ceramic Films, Acta Phys.Pol. A, 126(3), 811-819.

[10] J.P.Šetrajčić, D.Raković (1997) Fullerene C60 and Superconductivity - Fullerenes and Nanotubes Review, Vol.1, No.4, IHIS Fullerenes Science \& Technology Center, Beograd.

[11] J.P.Šetrajčić, M.Pantić, B.S.Tošić, D.Lj.Mirjanić (1995) Phonon States in Broken-Symmery Thin Films and Some Possible Consequences on Superconductivity, Bal.Phys.Lett., 2, 734-741.

[12] J.P. Šetrajčić, D.Lj. Mirjanić, I.J. Šetrajčić (2009) Charge Carriers Contribution in Ultrathin Film Thermodynamics and Superconductive Consequences, Proceedings UNITECH'09, 1-3.

[13] G.Rickayzen (1980) Green's Functions and Condensed Matter, Acad. Press, London.

[14] G.Mahan (1990) Many Particle Physics, Plenum Press, New York.

[15] C.Kittel (1963) Quantum Theory of Solids, Wiley \& Sons, New York.

[16] R.Simon (1991) High-Tc Thin Film and Electronic Devices, Physics Today, 44, 64-73.
[17] D.R.Harshman, A.P.Mills (1992) Concerning the Nature of High-Tc Superconductivity: Survey of Experimental Properties and Implications for Interlayer Coupling, Phys. Rev. B, 45, 10684-10691.

[18] W.E. Pickett (1989) Electronic Structure of the HighTemperature Oxide Superconductors, Rev. Mod. Phys., 61, 433-443.

[19] C.W.Chu (1995) Novel High Temperature Superconductor and High Superconductivity Physics, TICS'95, Hualien.

[20] J.P.Šetrajčić, B.S.Tošić, D.Lj.Mirjanić, S.Lazarev (1994) Estimates of The Effect of Electron(Hole)Phonon Interaction on Superconducting Characteristic of Thin Deformed Structures, Bal. Phys. Lett., 2, 741-744.

[21] D.Lj.Mirjanić, J.P.Šetrajčić (2002) The Nature of Superconducting State of Substance, Proceedings UNITECH'02, 78-81.

[22] J.P.Šetrajčić, B.S.Tošić, S.K.Jaćimovski, D.Lj. Mirjanić, S.M. Vučenović (2004) Electron Energies and Ordering in a Superconductive Ceramics, Proceedings UNITECH'04 3, 464-468.

[23] B.S.Tošić, V.D.Sajfert, S.K.Jaćimovski, J.P. Šetrajčić, D.I. Ilić, D.Lj. Mirjanić, S.M. Vučenović (2007) Electron-Phonon Interaction in Ultrathin Films and Superconductive Effects, Proceedings 17. SFKM, 72-75.

[24] D.Lj.Mirjanić, J.P.Šetrajčić (2009) Contemporary Supercondutivity Materials, Proceedings 1st International Congress: Engineering, Materials and Menangement in the Procesing Industry (EMMPI), 01-12.

[25] J.P.Šetrajčić, V.M.Zorić, N.V.Delić, D.Lj.Mirjanić, S.K. Jaćimovski (2011) Phonon Participation in Thermodynamics and Superconductive Properties of Thin Ceramic Films, Chapter 15, p. 317-348, In „Thermodynamics”, Ed. M. Tadashi, ISBN: 978-953307-544-0, InTech, Vien

\section{IZVOD}

\section{MOGUĆA STANJA NOSILACA NAELEKTRISANJA U TANKIM VIŠESLOJNIM SUPERPROVODNIM KERAMIKAMA}

$U$ radu je analizirano ponašanje (spektri $i$ stanja) elementarnih nosilaca naelektrisanja $u$ anizotropnim perovskitnim strukturama, kakve su savremene superprovodne keramike. Translaciona simetrija atomskih (jonskih) rasporeda sistema elektrona (ili šupljina) je narušena atomskim/jonskim/molekulskim rasprašivanjem (spaterovanjem) i dopiranjem, kao i postojanjem dveju graničnih površi. Ovo je model nosilaca naelektrisanja kod visoko-temperaturskih superprovodnika u kojem se posmatrano narušenje simetrije normalno na CuO ravni tretira kao perturbacija. Određene su jedno-čestične fermionske talasne funkcije i moguće energije nosilaca naelektrisanja.

Ključne riječi: Nosioci naelektrisanja, granice, anizotropija, energetska stanja i spektri, jednočestične talasne funkcije.

Naučni rad

Rad primljen: 09. 02. 2016.

Rad prihvaćen: 24. 03. 2016.

Rad je dostupan na sajtu: www.idk.org.rs/casopis 